\title{
The different roles of category- and feature-specific attentional control settings on attentional enhancement and inhibition
}

\author{
$\mathrm{Xia} \mathrm{Wu}^{1,2} \cdot$ Shimin $\mathrm{Fu}^{3}$
}

Published online: 6 July 2017

(C) The Psychonomic Society, Inc. 2017

\begin{abstract}
Contingent attentional capture suggests that topdown attentional control settings (ACS) can enhance attentional processing of task-relevant properties and inhibit attentional processing of task-irrelevant properties. However, it remains unclear how ACS operates when a distractor has both taskrelevant and task-irrelevant characteristics. In the present study, two lateralized ERP components, N2pc and distractor positivity (Pd), were employed as markers of attentional enhancement and inhibition, respectively. The degree of matching between a distractor and a conjunctively defined target was manipulated to illustrate attentional guidance by category-specific ACS (cACS) and feature-specific ACS (fACS), and the relative position between the distractor and the target was manipulated to isolate the processing of the distractor and the target. Experiment 1 showed that, with a long display duration for searching, a reliable N2pc component was elicited by a distractor that was feature-matched but category-mismatched $(\mathrm{C}-\mathrm{F}+)$ relative to the target-defined properties, suggesting an enhancing effect of fACS. In contrast, Experiment 2 demonstrated that, with a short display duration, a Pd component was elicited by a distractor that was feature-mismatched but category-matched $(\mathrm{C}+\mathrm{F}-)$ relative to the target-defined properties, suggesting an inhibitory effect of fACS. Moreover, both attentional enhancement and inhibition were only triggered by
\end{abstract}

Shimin $\mathrm{Fu}$

sfu@tsinghua.edu.cn; shimin.fu@gmail.com

1 Academy of Psychology and Behavior, Tianjin Normal University, Tianjin 300074, China

2 Center of Collaborative Innovation for Assessment and Promotion of Mental Health, Tianjin 300074, China

3 Department of Psychology, Tsinghua University, Beijing 100084, China
fACS but not by cACS. In summary, ACS can enhance targetrelevant properties or inhibit target-irrelevant properties in response to the display duration, and fACS affects both enhancement and inhibition more than cACS.

Keywords Attentional control settings $\cdot \mathrm{N} 2 \mathrm{pc} \cdot$ Distractor positivity $\cdot$ Inhibition $\cdot$ Enhancement $\cdot$ Category

A salient or singleton stimulus often elicits rapid and involuntary attentional allocation, which is referred to as attentional capture (Yantis \& Jonides, 1984). The bottom-up (stimulus-driven) account generally postulates that attentional capture is solely determined by sensory properties (Theeuwes, 1991; 2010; Hickey, McDonald, \& Theeuwes, 2006). However, the contingent involuntary orienting hypothesis (Folk, Remington, \& Johnston, 1992) suggests a top-down (goal-directed) view that attentional capture is determined by current top-down task sets (Serences et al., 2005). In a study by Folk et al. (1992), a spatial cueing effect indicative of attentional capture was observed only when the cue matched the current task, but it disappeared for a task-irrelevant cue, indicating the effect of top-down processing on attentional capture. Moreover, Folk et al. (1992) emphasized an attentional control setting (ACS), similar to an information filter, that determines which property is currently task-relevant and ensures attentional processing for matching objects or locations. For example, when the target was red, a red-specific ACS would prioritize the "red" property.

ACS has been observed for color, shape, movement, sudden onset, and even category (Atchley, Kramer, \& Hillstrom, 2000; Folk, Remington \& Wright, 1994; Folk, Leber, \& Egeth, 2008; Wyble, Folk, \& Potter, 2013; Wu, Liu, \& Fu, 2016). Although multiple ACSs have been demonstrated to 
operate simultaneously in complex and conjunction search tasks (Adamo, Pun, \& Ferber, 2010; Moore \& Weissman, 2010; Irons, Folk, \& Remington, 2012; Kiss, Grabert, \& Eimer, 2013), it is of interest to investigate the differences among ACSs. For instance, Wu et al. (2016) found that a category-specific ACS (cACS) and a feature-specific ACS (fACS) are activated independently at an early stage but are integrated at a later stage. Moreover, cACS and fACS were differently influenced by attentional engagement. Leblanc, Prime, and Jolicoeur (2008) showed that a relevant but not defined property (e.g., the digit category when searching for a red digit among gray digits) also could trigger categoryspecific attentional capture, indicating that ACS influences the processing of stimuli. Wu et al. (2013) found that when targets are defined at the category level, the feature-matching item can elicit an N2pc component, whereas activating a feature-specific template did not result in activation of the matching category. Therefore, it is worth investigating the relationship and differences between cACS and fACS by employing a conjunction search task.

Previous studies have reported an enhancing effect of ACS on target-relevant processing (Folk et al., 2008; Wyble et al., 2013). In addition, some studies have found an inhibitory effect of ACS on distractors during visual search (Hilimire, Hickey, \& Corballis, 2012; Sawaki, Geng, \& Luck, 2012). The N2pc component, a negative ERP difference that emerges approximately 200-ms poststimulus at electrode sites contralateral to the target relative to electrode sites ipsilateral to the target, often is utilized as an index of enhanced target attention (Eimer, 1996; Mazza, Turatto, \& Caramazza, 2009) or an index of active distractor inhibition (Luck \& Hillyard, 1994; Luck, Girelli, McDermott, \& Ford, 1997). Hickey, Di Lollo, and McDonald (2009) tried to isolate these two attentional processes of a target and a distractor by varying the relative positions between the target and the distractor. One target and one distractor were presented in three formations: a horizontal target and a vertical distractor (ThDv), a vertical target and a horizontal distractor (TvDh), and two contralateral stimuli (ThDh). With the premise that lateralized ERP activity could be elicited by stimuli presented in the horizontal meridian rather than in the vertical meridian (Woodman \& Luck, 2003), the results showed that target negativity (Nt) was elicited by the target under the ThDv condition, while distractor positivity $(\mathrm{Pd})$ was elicited by the distractor under the TvDh condition. Therefore, $\mathrm{Nt}$ and $\mathrm{Pd}$, reflecting the processing of target enhancement and distractor inhibition, respectively, are referred to as two subcomponents of the N2pc elicited under the $\mathrm{ThDh}$ condition (i.e., the traditional stimuli presentation method used in previous studies; Eimer, 1996; Luck \& Hillyard, 1994).

However, it remains unknown whether a distractor that shares target-defined properties would elicit an N2pc, indicative of enhancement for target-relevant properties, or a $\mathrm{Pd}$, indicative of inhibition for target-irrelevant properties. Kiss, Grubert, Petersen, and Eimer (2012) showed that the display durations of a visual search task might trigger different processing of a distractor. Specifically, when the display durations were short, the distractor elicited a reliable N2pc, indicating an enhancing effect of ACS, whereas the distractor elicited a reliable Pd with short display durations, indicating an inhibitory effect of ACS. Notably, in Kiss et al. (2012), the target was only defined by a shape, such that the distractorelicited N2pc would be inferred as an effect of bottom-up salience, but not an effect of a target-relevant property. The influence of display durations on the effects of target-relevant and target-irrelevant properties remains unclear.

The purpose of the present study was to investigate the different roles of $\mathrm{CACS}$ and AACS on attentional enhancement and inhibition by a distractor. First, to isolate cACS and fACS, we employed a target that was defined by the combination of a category $(\mathrm{C}$, letter or digit) and a feature ( $\mathrm{F}$, red or blue). The degree of matching between the distractor and the target were manipulated as $\mathrm{C}+\mathrm{F}-, \mathrm{C}-\mathrm{F}+$, and $\mathrm{C}-\mathrm{F}$ - so that the individual effects of cACS and fACS could be revealed. Second, to isolate the processing of the distractor from the target, we adopted a visual search array containing one target and one distractor, similar to Hickey et al. (2009). Importantly, lateralized ERP activity was indicative of a distractor when the target was located at a vertical position and the distractor was located at a horizontal meridian (TvDh). Moreover, data for three relative positions between the target and the distractor (ThDv, ThDh, TvDv) were recorded. Third, to investigate the impact of the enhancing and inhibitory effects of ACSs for a distractor, we conducted two experiments with different display durations. An N2pc indicative of attentional enhancement was assumed to be elicited by a distractor with long display durations (1,000 $\mathrm{ms}$ in Experiment 1), and a $\mathrm{Pd}$ indicative of attentional inhibition was assumed to be elicited by a distractor with short display durations (300 ms in Experiment 2).

\section{Experiment 1}

\section{Methods}

\section{Participants}

Eighteen volunteers recruited from Tsinghua University Forum participated for payment. Three of them were excluded from analyses because of excessive eye movements and alpha activity. The remaining 15 participants (10 males) ranged in age from 18 to 33 years $(M=22.9$, $S D=1.2$ ). All had self-reported normal or corrected-tonormal visual acuity and color vision. 


\section{Experiment 1}

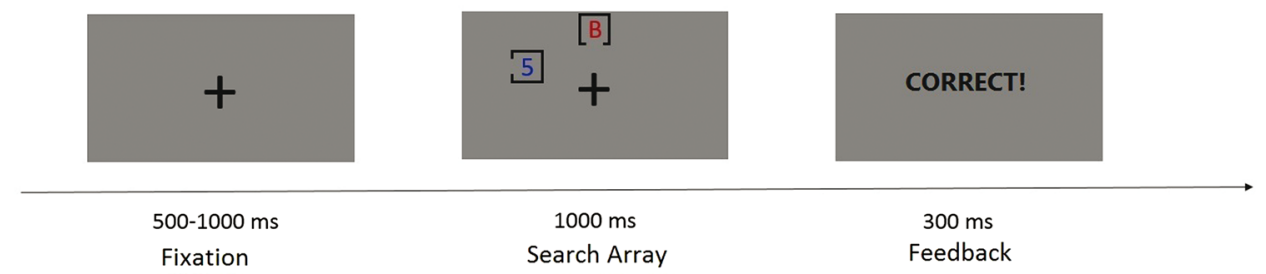

\section{Experiment 2}
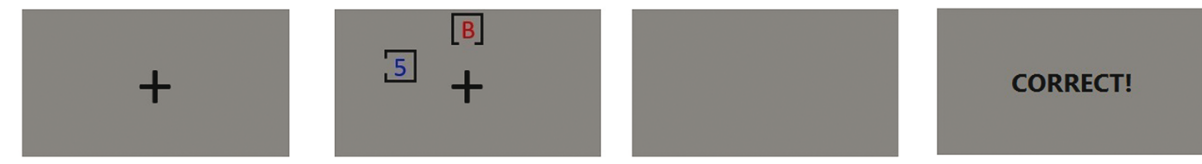

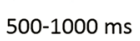

Fixation

Search Array

Fig. 1 Procedure of Experiments 1 and 2. Participants were instructed to report the orientation of the frame gap surrounding the target (red letter). The target was defined by the combination of a feature (red or blue) and a category (letter or digit), which corresponded to a red letter, a blue letter, a red digit or a blue digit. These four combinations of target-defining properties were counterbalanced across participants. Two factors were manipulated, including the relative positions between the target and the

\section{Stimuli and procedure}

Stimuli were presented on a CRT monitor with a refresh rate of $100 \mathrm{HZ}$ and a resolution of $1024 \times 768$ pixels (Fig. 1). The participants were seated in a dimly lit room at a distance of approximately $57 \mathrm{~cm}$ from the monitor. Stimulus presentation and response collection were controlled using E-Prime software (version 2.0). Each search array consisted of two symbols placed around a central fixation cross. Each symbol subtended $1.1^{\circ} \times 0.8^{\circ}$ and enclosed a black surrounding frame $\left(1.5^{\circ} \times 1.4^{\circ}\right)$ whose gap was randomly assigned to up, down, left and right. The symbol in the search array could be a letter (except I, $\mathrm{J}, \mathrm{O}, \mathrm{Q})$ or digit (2-9) shown in red (RGB, 145, 0, 0) or blue (RGB, 0, 0, 255) according to different conditions. Both colors were equiluminant $\left(13 \mathrm{~cd} / \mathrm{m}^{2}\right)$, and all stimuli were presented against a gray (RGB, 128, 128, 128) background.

Each trial began with a fixation point presented randomly between 500 and $1000 \mathrm{~ms}$, followed by a search array presented for 1,000 ms. Two symbols in search array appeared randomly with equiprobably at one of the six possible locations around the fixation point at a radial distance of $3.5^{\circ}$. Two positions were on the vertical meridian while the other four positions were located at $60^{\circ}, 120^{\circ}, 240^{\circ}$, and $300^{\circ}$ from vertical. Participants were instructed to report the orientation of the frame gap surrounding the target by pressing the arrow keys on the keyboard, as fast and accurately as they could. The target was defined by the combination of a feature (red or blue) and a category (letter or digit), which corresponded to a distractor $(\mathrm{ThDv}, \mathrm{TvDh}, \mathrm{ThDh}, \mathrm{TvDv} ; \mathrm{T}=$ target, $\mathrm{D}=$ distractor, $\mathrm{h}=$ horizontal, $\mathrm{v}=$ vertical; $\mathrm{TvDh}$ is shown in this figure) and the degrees of matching levels between the distractor and the target $(\mathrm{C}+\mathrm{F}-, \mathrm{C}-\mathrm{F}+, \mathrm{C}$ $\mathrm{F}-; \mathrm{C}=$ category, $\mathrm{F}=$ feature, $+=$ match, $-=$ mismatch C-F- is shown in this figure). The only difference between two experiments was the display durations of search array $(1,000 \mathrm{~ms}$ in Experiment 1, the above panel; $300 \mathrm{~ms}$ in Experiment 2, bottom panel)

red letter, a blue letter, a red digit or a blue digit, yielding a conjunction search task. These four target-defining properties were counterbalanced across participants. To amplify the effect of ACS, ${ }^{1}$ in one sixth of all trials, a probe condition was included in which no target (two distractors) appeared and a SPACE response was required. In the remaining trials, the search array consisted of one target and one distractor that were manipulated by two independent factors, the degree of matching and the relative position between the distractor and the target. Specifically, the degree of matching corresponded to $\mathrm{C}+\mathrm{F}-, \mathrm{C}-\mathrm{F}+$, or C-F- for category matching $(\mathrm{C})$ and feature matching $(\mathrm{F})$ between the distractor and the target. For example, if a red letter was the target, a blue letter, a red digit, and a blue digit were denoted $\mathrm{C}+\mathrm{F}-, \mathrm{C}-\mathrm{F}+$, and $\mathrm{C}-\mathrm{F}-$, respectively. The relative positions were set to $\mathrm{ThDv}, \mathrm{TvDh}, \mathrm{ThDv}$, or TvDv, representing the relative horizontal (h) and vertical (v) positions of the target (T) and the distractor (D). Feedback for $300 \mathrm{~ms}$ was presented at the end of each trial. The entire experiment comprised 10 practice trials followed by 96 trials for each $3 \times 4$ condition for a total of 1,162 trials (192 trials for probe condition). Participants were allowed a 1-

\footnotetext{
${ }^{1}$ In a preliminary experiment, some participants reported using a strategy to find the difference between two stimuli, rather than finding the target-defining property. The participants focused on color-matching if the two stimuli were colored differently, decreasing the effect of cACS. Similarly, a strategy of focusing on category matching would decrease the effect of fACS. To avoid this strategy, we added a probe condition to force the participant to detect the fully matching stimulus, rather than to first focus on the difference between the target and the distractor.
} 
minute rest after every 20 trials, and thus, the experiment lasted approximately 75 minutes.

\section{Electrophysiological recording and analysis}

Electroencephalograms (EEGs) were recorded from 64 scalp sites at standard positions of the extended International 10-20 system (Quikcap, Compumedics, North Carolina, USA). EEGs were sampled at $500 \mathrm{~Hz}$ with a $40-\mathrm{Hz}$ low-pass filter. During recording, all electrodes were referenced to the reference site (located halfway between $\mathrm{CPz}$ and $\mathrm{Pz}$ ) and were rereferenced offline to the average of the left and right mastoids. Continuous EEG was segmented from -200 to 800 ms relative to the onset of the search array. Trials with artifacts (vEOG exceeding $\pm 60 \mu \mathrm{V}, \mathrm{hEOG}$ exceeding $\pm 25 \mu \mathrm{V}$, all other channels exceeding $\pm 75 \mu \mathrm{V}$ ) were removed before averaging. The waveforms with correct responses were averaged separately for each combination of matching (C+F-, C-F+, C-F-) and position (ThDv, TvDh, ThDh, TvDv). The lateral components elicited by the target or the distractor were quantified by the mean ERP amplitude in an appropriate time window (220$280 \mathrm{~ms})^{2}$ following the onset of the search array at posterior lateral electrodes PO7 and PO8. Specifically, the waveforms observed at PO7 when the item was presented at the upper right or the lower right were averaged with the waveform observed at PO8 when the item was presented at the upper left or the lower left, yielding the contralateral waveforms. In contrast, the waveforms observed at PO8 when the item was presented at the upper right or the lower right were averaged with the waveform observed at $\mathrm{PO} 7$ when the item was presented at the upper left or the lower left, yielding the ipsilateral waveforms. Difference waveforms were obtained by subtracting the ipsilateral waveforms from the contralateral waveforms. ${ }^{3}$ Greenhouse-Geisser corrections were used where appropriate. The differences between conditions were assessed with two-tailed $t$ tests.

\section{Results}

\section{Behavioral results}

The mean accuracy and RTs for the probe condition were $77.4 \%(S D=3.0 \%)$ and $623 \mathrm{~ms}(S D=7.9)$, respectively. The

\footnotetext{
2 The time window for the contralateral component was selected based on the individual $\mathrm{N} 2 \mathrm{pc}$ maximum reflection for each condition and participant (we did not show a large number of laterality figures due to the length of the article), which generally peaked at $250 \mathrm{~ms}$ after the search display. Then, a 30-ms range was applied before and after the peak, in response to the beginning and ending latency of contralateral differences. We averaged all the data after selecting the time window, and the consistent results between Experiments 1 and 2 suggested the validity of this time window.

${ }^{3}$ In the TvDv condition, upper stimuli were considered as the left stimuli and lower stimuli were considered as the right stimuli when subtracting and producing the contralateral components.
}

mean accuracies of target identification (Table 1) were subjected to a repeated-measures ANOVA with matching $(\mathrm{C}+\mathrm{F}-, \mathrm{C}-\mathrm{F}+$ , C-F-) and position (ThDv, TvDh, ThDh, TvDv) factors. The analysis revealed a main effect of matching $[F(2,28)=14.80$, $M S E=0.026, p<0.005]$, a main effect of position $[F(3,42)=$ 3.56, MSE $=0.001, p<0.05]$, and an interaction between matching and position $[F(6,84)=2.88, M S E=0.002, p<$ 0.05]. Multiple comparisons of the degrees of matching revealed that the accuracy was significantly lower under the $\mathrm{C}$ $\mathrm{F}+$ condition than under the C+F- $(p<0.01)$ and C-F- $(p<$ $0.005)$ conditions. Multiple comparisons of the positions revealed that the accuracy was marginally significantly lower under the TvDv condition than under the $\operatorname{ThDh}(p=0.06)$ condition. To further explore the interaction, a simple main effects analysis of matching was conducted for each position, which revealed a lower accuracy under $\mathrm{C}-\mathrm{F}+$ than under $\mathrm{C}+\mathrm{F}-$ and C-F- for all four positions ( $p s<0.05$ ).

The repeated-measures ANOVA for the RTs of target identification (Table 1) revealed a main effect of matching $[F(2,28)=$ $110.35, M S E=1739.167, p<0.001]$. Multiple comparisons of the degree of matching showed a similar lower performance (longer RT) for target under $\mathrm{C}-\mathrm{F}+$ than under $\mathrm{C}+\mathrm{F}-(p<$ $0.001)$ and C-F- $(p<0.001)$. The consistent result of accuracy and RT suggest that the feature-matching distractor $(\mathrm{C}-\mathrm{F}+)$ can capture attention and interfere with the processing of target identification.

\section{ERP results}

Figure 2 shows the grand-average ERP waveforms elicited within $350 \mathrm{~ms}$ after search array onset at electrodes PO7/ PO8 as a function of the degree of matching (columns) and relative position (rows). Solid and dashed lines show ERPs contralateral and ipsilateral to the location of the distractor, respectively. The left column of Figure 4 shows the difference waves across each condition. Repeated-measures ANOVA for the mean amplitudes between 220 and $280 \mathrm{~ms}$, with factors of laterality (contralateral and ipsilateral), matching (C+F-, C-F+ , C-F-), and position (ThDv, TvDh, ThDh, TvDv) was performed. The analysis revealed a main effect of laterality $[F(1$, $14)=35.92, M S E=2.412, p<0.001]$, which indicated a significantly negative wave at the contralateral side relative to the ipsilateral side, indicating reliable contralateral components. The main effect of matching also was significant $[F(2$, 28) $=10.99, M S E=0.957, p<0.001]$, which revealed that the ERPs corresponding to $\mathrm{C}+\mathrm{F}$ - and $\mathrm{C}-\mathrm{F}+$ were significantly more negative than those corresponding to C-F- $(p s<0.005)$ according to multiple comparisons. In addition, a main effect of position was also significant $[F(3,42)=10.13, M S E=$ $3.319, p<0.001]$, which showed the waves under the TvDv condition were significantly more negative than under the other three positions $(p s<0.05)$. Moreover, there were interactions of laterality $\times$ matching $[F(2,28)=4.63, M S E=$ 
Table 1 Accuracy (ACC) and Reaction times (RTs) of Correct Reports in Experiment 1 and Experiment 2 (Mccu)

\begin{tabular}{|c|c|c|c|c|c|c|c|}
\hline & & \multicolumn{3}{|c|}{$\begin{array}{l}\text { Experiment } 1 \\
\text { (1000 ms display duration) }\end{array}$} & \multicolumn{3}{|c|}{$\begin{array}{l}\text { Experiment } 2 \\
\text { (300 ms display duration) }\end{array}$} \\
\hline & & $\mathrm{C}+\mathrm{F}-$ & $\mathrm{C}-\mathrm{F}+$ & C-F- & $\mathrm{C}+\mathrm{F}-$ & $\mathrm{C}-\mathrm{F}+$ & C-F- \\
\hline \multirow[t]{2}{*}{ ThDv } & $A C C$ & $.98 \pm .02$ & $.88 \pm .12$ & $.98 \pm .03$ & $.98 \pm .03$ & $.87 \pm .09$ & $.97 \pm .02$ \\
\hline & $R T s$ & $574 \pm 53$ & $644 \pm 49$ & $569 \pm 50$ & $562 \pm 60$ & $644 \pm 55$ & $559 \pm 55$ \\
\hline \multirow[t]{2}{*}{ TvDh } & $A C C$ & $.98 \pm .02$ & $.89 \pm .10$ & $.99 \pm .02$ & $.97 \pm .03$ & $.87 \pm .07$ & $.98 \pm .02$ \\
\hline & $R T S$ & $574 \pm 53$ & $647 \pm 41$ & $575 \pm 51$ & $564 \pm 58$ & $654 \pm 47$ & $564 \pm 54$ \\
\hline \multirow[t]{2}{*}{ ThDh } & $A C C$ & $.98 \pm .02$ & $.90 \pm .10$ & $.98 \pm .02$ & $.98 \pm .02$ & $.87 \pm .09$ & $.98 \pm .02$ \\
\hline & $R T S$ & $572 \pm 47$ & $647 \pm 49$ & $572 \pm 50$ & $560 \pm 56$ & $631 \pm 51$ & $564 \pm 59$ \\
\hline \multirow[t]{2}{*}{ TvDv } & $A C C$ & $.98 \pm .02$ & $.85 \pm .10$ & $.98 \pm .02$ & $.98 \pm .03$ & $.81 \pm .09$ & $.97 \pm .03$ \\
\hline & $R T s$ & $574 \pm 43$ & $651 \pm 30$ & $575 \pm 43$ & $564 \pm 57$ & $649 \pm 37$ & $560 \pm 56$ \\
\hline
\end{tabular}

$0.539, p<0.05]$, laterality $\times$ position $[F(3,42)=25.94, M S E=$ $1.112, p<0.001]$, and laterality $\times$ matching d lateralit $[F(6,84)$ $=10.03, M S E=0.666, p<0.001]$. Follow-up $t$ tests comparing contralateral and ipsilateral ERPs were conducted separately on every matching $\times$ position condition. Under the
ThDv condition, a reliable $\mathrm{Nt}$ was observed for $\mathrm{C}+\mathrm{F}-[t(14)$ $=-6.35, p<0.001], \mathrm{C}-\mathrm{F}+[t(14)=-3.87, p<0.005]$ and C-F$[t(14)=-6.70, p<0.001]$, suggesting that the target could trigger reliable early attention allocation. Under the TvDh condition, a reliable distractor-N2pc was only observed on $\mathrm{C}-\mathrm{F}+[t$ a

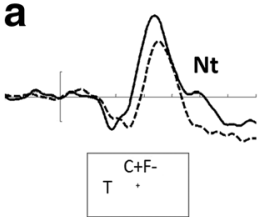

b

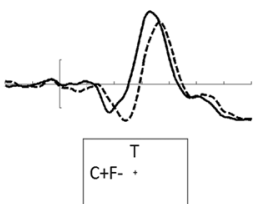

C

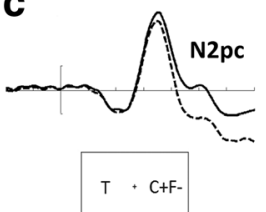

d

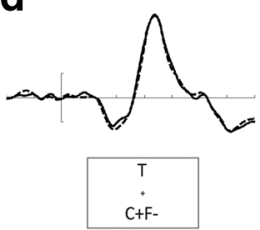

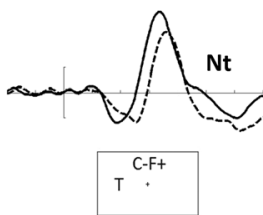
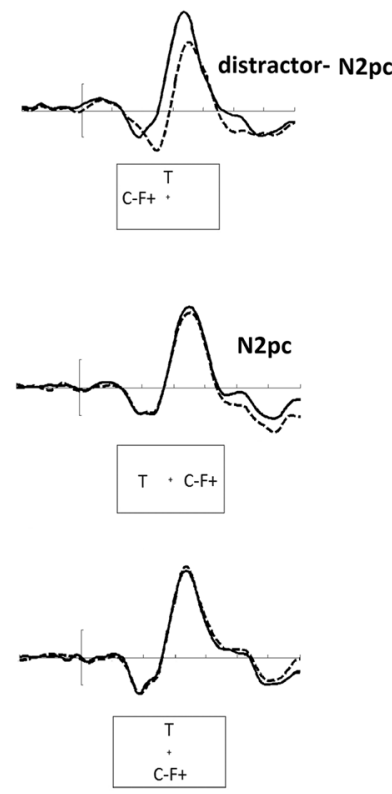

Contralateral to target Ipsilateral to target

Fig. 2 Grand-averaged ERPs elicited in Experiment 1 for each degree of matching conditions $(\mathrm{C}+\mathrm{F}-, \mathrm{C}-\mathrm{F}+, \mathrm{C}-\mathrm{F}-$, shown in left, middle, and right columns, respectively) under four relative positions ( $\mathrm{ThDv}, \mathrm{TvDh}, \mathrm{ThDh}$, TvDv, shown in A, B, C, and D, respectively). These ERPs were recorded at electrode sites PO7 and PO8 contralateral (solid lines) and ipsilateral
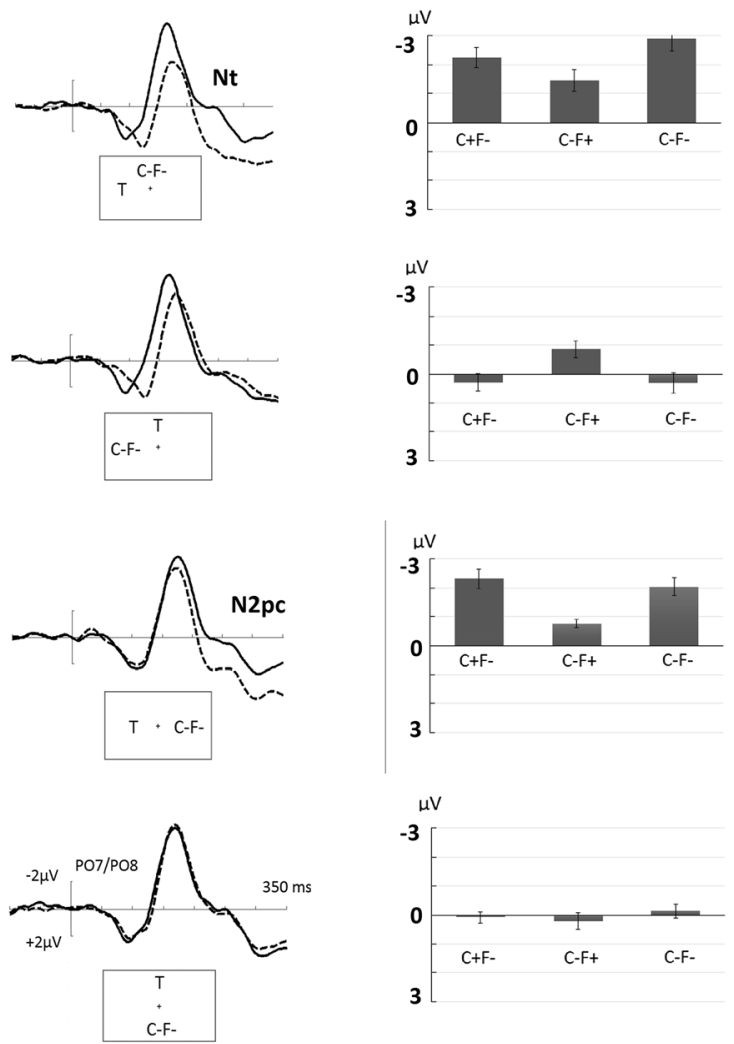

(dashed lines). These represent differences between brain activity measured in 220-280 ms time window after search array onset over hemispheres ipsilateral and contralateral to lateral stimuli, and the mean amplitudes of contralateral difference waves were shown in rightmost bar graph 
$(14)=-2.95, p<0.02]$, suggesting that fACS could enhance the processing of the target-relevant property of the distractor. Under the ThDh condition, a reliable N2pc was observed on $\mathrm{C}+$ $\mathrm{F}-[t(14)=-6.96, p<0.001], \mathrm{C}-\mathrm{F}+[t(14)=-4.93, p<0.001]$ and C-F- $t(14)=-6.54, p<0.001]$. Under the TvDv condition, no lateralized posterior ERPs were observed ( $p s>0.48$ ). To clearly explore the effects of matching and position, the mean magnitude of the lateralized component measured by subtracting ipsilateral waveforms from contralateral waveforms across a time window of 220-280 ms after the onset of the search array was performed for further analysis.

Repeated-measures ANOVA for the mean magnitude of the contralateral component with matching $(\mathrm{C}+\mathrm{F}-, \mathrm{C}-\mathrm{F}+, \mathrm{C}-\mathrm{F}-)$ and position (ThDv, TvDh, ThDh, TvDv) factors was performed. The analysis revealed a main effect of matching $[F(2,28)=$ 4.63, $M S E=1.079, p<0.05]$, a main effect of position $[F(3$, $42)=25.94, M S E=2.225, p<0.001]$, and an interaction between matching and position $[F(6,84)=10.03, M S E=1.333$, $p<0.001]$. To further explore the interaction, simple effects analyses for matching were conducted under each position. Under ThDv, a smaller Nt was elicited by the target when it was presented with the $\mathrm{C}-\mathrm{F}+$ distractor than with the $\mathrm{C}+\mathrm{F}-(p<$ $0.02)$ or C-F- $(p<0.005)$ distractor. Under TvDh, a larger N2pc was elicited by the $\mathrm{C}-\mathrm{F}+$ distractor than by the $\mathrm{C}+\mathrm{F}-(p<0.001)$ and C-F- $(p<0.02)$ distractors. Under ThDh, a similar larger $\mathrm{N} 2 \mathrm{pc}$ was elicited by the target when it was presented with the $\mathrm{C}-\mathrm{F}+$ distractor than with the $\mathrm{C}+\mathrm{F}-$ or $\mathrm{C}-\mathrm{F}-(p s<0.005)$ distractors. These simple effects results could indicate that fACS plays a critical role relative to $\mathrm{cACS}$ in enhancing the processing of corresponding target-relevant properties.

Experiment 1 was conducted to explore the effect of cACS and fACS on distractors within long display durations. The critical finding was that fACS could operate on distractors and enhance the processing of target-relevant properties. Specifically, when the search array contained a vertical target and a horizontal distractor (TvDh), an N2pc component rather than a $\mathrm{Pd}$ was elicited by a feature-matching distractor $(\mathrm{C}-\mathrm{F}+)$, suggesting that the distractor captured attention. However, the lateralized ERP components elicited by a category-matching distractor $(\mathrm{C}+\mathrm{F}-)$ were equivalent to those elicited by the purely salient distractor $(\mathrm{C}$ F-), suggesting that the effects of cACS eliminated or even disappeared in this conjunction search task.

\section{Experiment 2}

\section{Methods}

\section{Participants}

Twenty volunteers (14 males) recruited from Tsinghua University Forum participated for payment (age range 19-28 years; $M=22.0, S D=0.8$ ). All had self-reported normal or corrected-to-normal visual acuity and color vision.

Apparatus, stimuli, procedure, design, EEG recording, and analysis

Apparatus, stimuli, procedure, design, EEG recording, and analysis were identical to the Experiment 1, except that the search array were presented for only $300 \mathrm{~ms}$. Furthermore, a 700-ms black following the search array was presented to uniform the whole duration of each trial with the Experiment 1.

\section{Results}

\section{Behavioral results}

The mean accuracy and RTs for the probe condition were $85.0 \%(S D=2.8 \%)$ and $626 \mathrm{~ms}(S D=10.0)$, respectively. The mean accuracies of target identification (Table 1) were subjected to a repeated-measures ANOVA with matching (C+F-, C-F+, C-F-) and position (ThDv, TvDh, ThDh, TvDv). The analysis revealed a main effect of the degrees of matching $[F(2,38)=67.03, M S E=0.011, p<0.001]$, a main effect of position $[F(3,57)=8.10, M S E=0.001, p<0.001]$ and the interaction between matching and position $[F(6,114)$ $=6.91, M S E=0.002, p<0.001]$. Multiple comparisons of levels of matching revealed that the accuracy was significantly lower under the $\mathrm{C}-\mathrm{F}+$ condition than that under the $\mathrm{C}+\mathrm{F}$ - and C-F- ( $p s<0.001)$ conditions. Multiple comparisons of levels of position revealed that the accuracy was significantly lower under the TvDv than that under the ThDh, TvDh, and ThDv conditions $(p s<0.01)$. A simple main effect of matching were conducted for each positions, which revealed a better performance of target under $\mathrm{C}-\mathrm{F}+$ than that under $\mathrm{C}+\mathrm{F}$ - and $\mathrm{C}-\mathrm{F}$ - for every position ( $p s<0.001)$, similar as Experiment 1 .

RT (Table 1) analyses showed a main effect of matching $[F$ $(2,38)=160.19, M S E=2004.806, p<0.001]$ and an interaction between matching and position $[F(6,114)=3.35, M S E=$ 285.286, $p<0.05$ ]. Multiple comparisons of levels of matching revealed that RTs were significantly longer under the $\mathrm{C}-\mathrm{F}+$ than that under the $\mathrm{C}+\mathrm{F}-$ and $\mathrm{C}-\mathrm{F}-(p s<0.001)$. A simple main effect of matching were conducted for each position, which showed that in all four positions, the RTs under $\mathrm{C}-\mathrm{F}+$ were significantly longer than that under $\mathrm{C}+\mathrm{F}-$ and $\mathrm{C}-\mathrm{F}-$ $(p s<0.001)$.

\section{ERP results}

Figure 3 shows grand-average ERP waveforms elicited in the $350 \mathrm{~ms}$ after search array onset at electrodes PO7/PO8 as a function of matching (columns) and relative positions (rows). Solid and dashed lines show ERPs contralateral and ipsilateral to the location of the distractor, respectively. The right column 
a

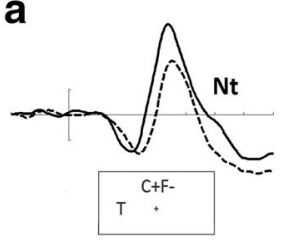

b

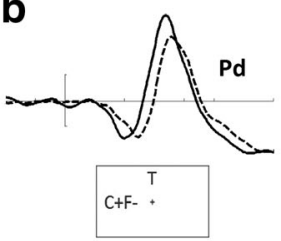

C

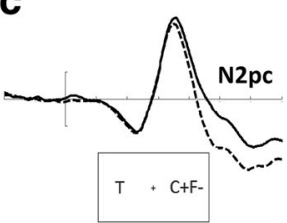

d

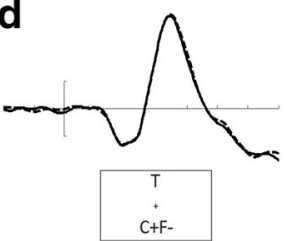

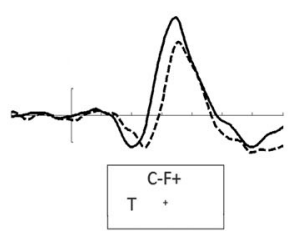
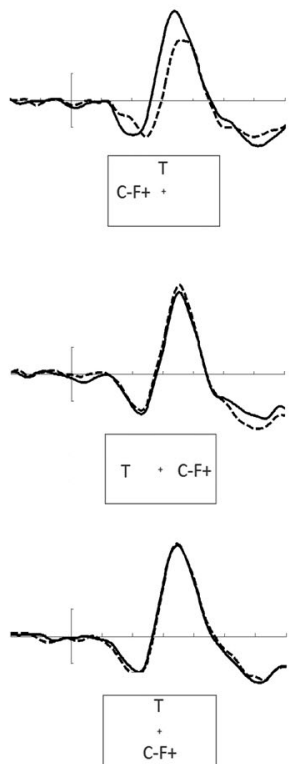

Contralateral to target Ipsilateral to target

Fig. 3 Grand-averaged ERPs elicited in Experiment 2 for each degree of matching conditions $(\mathrm{C}+\mathrm{F}-, \mathrm{C}-\mathrm{F}+, \mathrm{C}-\mathrm{F}-$, shown in left, middle, and right columns, respectively) under four relative positions ( $\mathrm{ThDv}, \mathrm{TvDh}, \mathrm{ThDh}$, TvDv, shown in A, B, C, and D, respectively). These ERPs were recorded at electrode sites PO7 and PO8 contralateral (solid lines) and ipsilateral

of Figure 4 shows the difference in waves across each condition. Repeated-measures ANOVA for the mean amplitudes at each area between 220 and $280 \mathrm{~ms}$, with factors of laterality (contralateral and ipsilateral), matching $(\mathrm{C}+\mathrm{F}-, \mathrm{C}-\mathrm{F}+, \mathrm{C}-\mathrm{F}-)$ and position (ThDv, TvDh, ThDh, TvDv) was performed. The analysis revealed a main effect of laterality $[F(1,19)=$ $8.17, M S E=5.725, p<0.01]$, corresponding to a significantly negative wave at the contralateral side relative to the ipsilateral side, indicating reliable contralateral components. There also was a main effect of matching $[F(2,38)=5.03, M S E=2.060$, $p<0.02]$, a main effect of position $[F(3,57)=12.28, M S E=$ $2.880, p<0.001]$ and interactions of laterality $\times$ matching $[F(2,38)=8.03, M S E=0.592, p<0.001]$, laterality $\times$ position $[F(3,57)=20.99, M S E=2.594, p<0.001]$ and laterality $\times$ matching $\times$ position $[F(6,114)=7.75, M S E=1.511, p<$ $0.001]$. Follow-up $t$ tests comparing contralateral and ipsilateral ERPs were conducted separately for each condition. Under the ThDv condition, a reliable $\mathrm{Nt}$ was observed for $\mathrm{C}+\mathrm{F}-[t(19)=-4.59, p<0.001]$ and C-F- $[t(19)=-3.47, p$ $<0.005$ ]. Under the TvDh condition, a reliable Pd was observed for $\mathrm{C}+\mathrm{F}-[t(19)=2.75, p<0.02]$ and C-F- $[t(19)=$
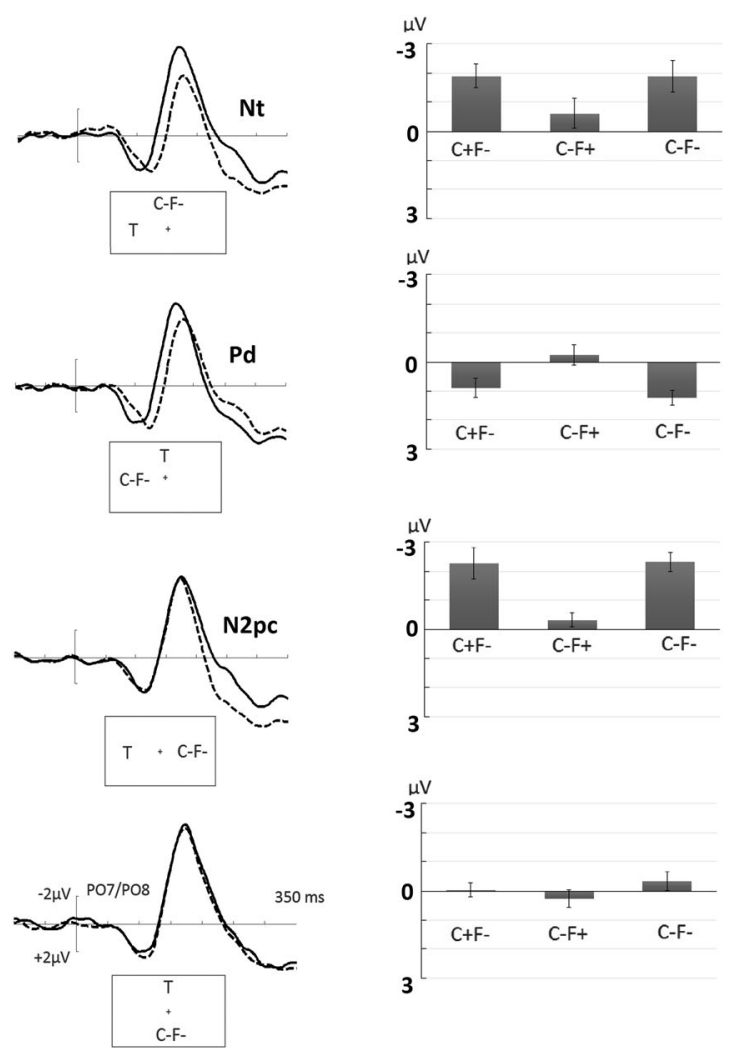

(dashed lines). These represent differences between brain activity measured in 220-280 ms time window after search array onset over hemispheres ipsilateral and contralateral to lateral stimuli, and the mean amplitudes of contralateral difference waves were shown in rightmost bar graph

$4.75, p<0.001]$, suggesting that ACS could inhibit the processing of target-irrelevant properties of the distractor. Under the ThDh condition, a reliable $\mathrm{N} 2 \mathrm{pc}$ was observed for $\mathrm{C}+\mathrm{F}-[t$ $(19)=-4.12, p<0.001]$ and C-F- $[t(19)=-6.95, p<0.001]$. Under the TvDv condition, no lateralized posterior ERPs were observed ( $p s>0.32$ ). To clearly explore the effects of matching and position, the mean magnitude of the lateralized component measured by subtracting ipsilateral waveforms from contralateral waveforms across a time window of 220$280 \mathrm{~ms}$ after the onset of the search array was performed for further analysis.

Repeated-measures ANOVA for the mean magnitude of the contralateral component with matching $(\mathrm{C}+\mathrm{F}-, \mathrm{C}-\mathrm{F}+, \mathrm{C}-$ $\mathrm{F}-$ ) and position ( $\mathrm{ThDv}, \mathrm{TvDh}, \mathrm{ThDh}, \mathrm{TvDv}$ ) factors was performed. The analysis revealed a main effect of matching $[F(2$, 38) $=8.03, M S E=1.184, p<0.001]$, a main effect of position $[F(3,57)=20.99, M S E=5.188, p<0.001]$, and an interaction between matching and position $[F(6,114)=7.15, M S E=$ 3.022, $p<0.001]$. Multiple comparisons of the degree of matching revealed that a larger contralateral component was elicited by $\mathrm{C}+\mathrm{F}-$ and $\mathrm{C}-\mathrm{F}-$ than by $\mathrm{C}-\mathrm{F}+(p<0.02$ and $p<$ 


\section{Experiment 1 (1000 ms) Experiment 2 (300 ms)}

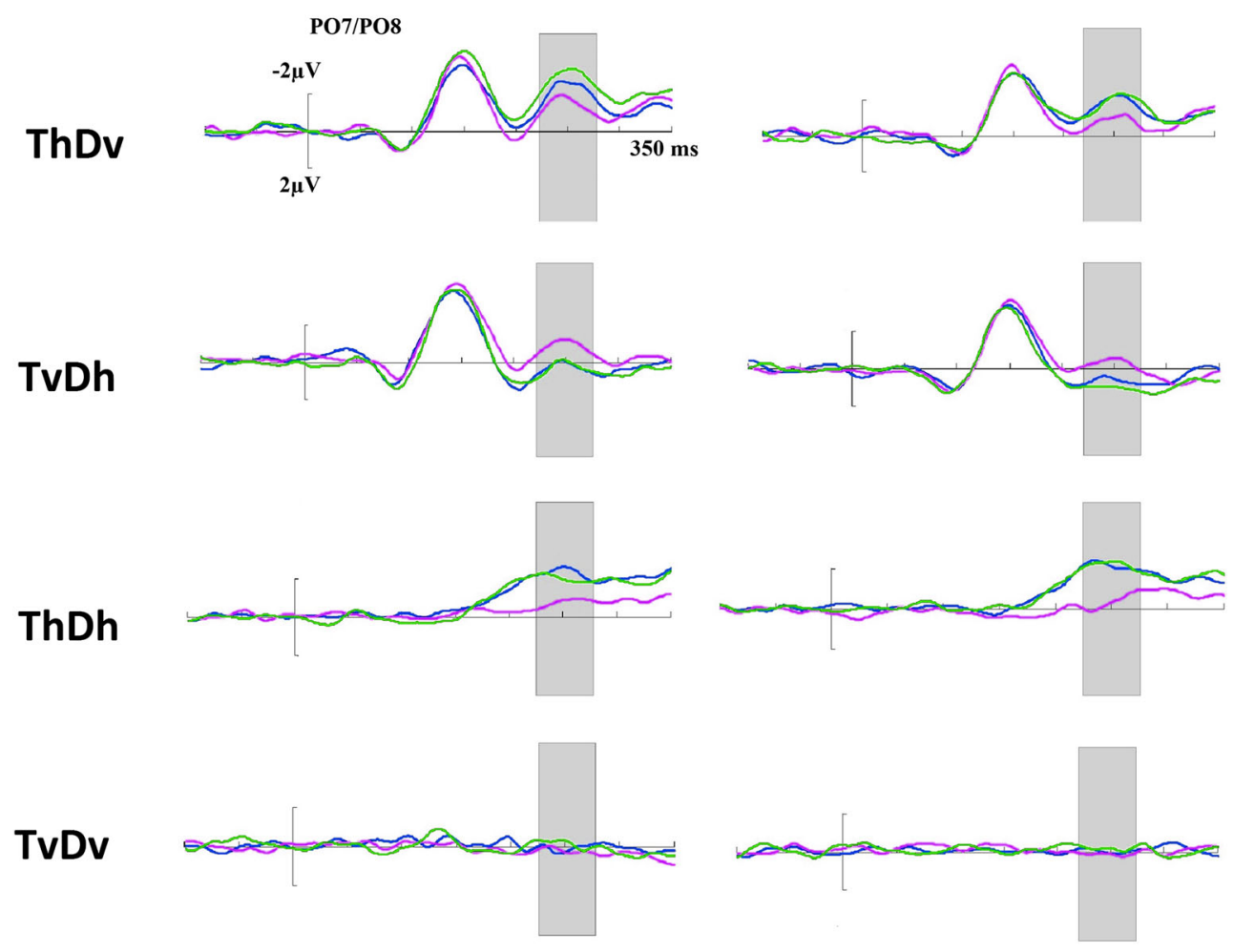

\section{$\mathrm{C}-\mathrm{F}+\longrightarrow \mathrm{C}+\mathrm{F}-\longrightarrow \mathrm{C}-\mathrm{F}-$}

Fig. 4 Different waveforms produced by subtracting ipsilateral from contralateral ERPs for each matching $\times$ position condition in Experiment 1 (left) and Experiment 2 (right). Gray shadows indicate the 220-280 ms time window

0.05 , respectively). To further explore the interaction, simple effects analyses of matching were conducted under each position. Under $\mathrm{ThDv}$, a smaller $\mathrm{Nt}$ was elicited by the target when it was presented with the $\mathrm{C}-\mathrm{F}+$ distractor than with the $\mathrm{C}+\mathrm{F}-(p<0.02)$ and C-F- $(p<0.001)$ distractors. Under TvDh, a smaller Pd was elicited by the C-F+ distractor than by the $\mathrm{C}+$ F- $(p<0.02)$ and C-F- $(p<0.001)$ distractors. Under ThDh, a similar larger $\mathrm{N} 2 \mathrm{pc}$ was elicited by the target when it was presented with the $\mathrm{C}-\mathrm{F}+$ distractor than with the $\mathrm{C}+\mathrm{F}-$ and C-F- ( $p s<0.005)$ distractors. These simple effects results could suggest an important role of fACS relative to cACS in inhibiting the processing of target-irrelevant properties.

Experiment 2 was conducted to explore the effect of cACS and AACS on the distractor within a short display duration. The key finding was that fACS could operate on the distractor and inhibit the processing of its target-irrelevant properties. Specifically, when the search array contained a vertical target and a horizontal distractor (TvDh), a Pd component rather than an $\mathrm{N} 2 \mathrm{pc}$ was elicited by the feature-mismatching distractor $(\mathrm{C}+\mathrm{F}-)$, indicating active inhibition of the distractor.
However, the lateralized ERP components elicited by the category-mismatching distractor $(\mathrm{C}-\mathrm{F}+)$ were equivalent to those of the purely salient distractor (C-F-), suggesting that the effects of cACS eliminated or even disappeared in this conjunction search task.

To further investigate whether the pattern of the lateralized component triggered by the distractor is determined by the display duration in the conjunction search task, an RANOVA with matching (C+F-, C-F+, C-F-) and position (ThDv, TvDh, $\mathrm{ThDh}, \mathrm{TvDv})$ as within-subjects factors and display duration as between-subjects factor (long durations in Experiment 1 vs. short durations in Experiment 2) was performed. The main effects of matching $[F(2,66)=11.52, p<0.001]$ and position $[F$ $(3,99)=42.08, p<0.001]$ and the interaction between matching $\times$ position $[F(6,198)=14.69, p<0.001]$ were observed. However, a main effect of duration across experiments $[F(1$, $33)=1.52, p=0.227]$ and interactions of matching $\times$ durations $[F(2,66)=0.60, p=0.55]$, position $\times$ durations $[F(3,99)=1.59$, $p=0.20]$ and matching $\times$ position $\times$ durations $[F(6,198)=0.48$, $p=0.82]$ were not found. 


\section{Discussion}

We conducted two ERP experiments to explore the different roles of CACS and fACS on attentional enhancement and inhibition of a distractor. The degree of matching $(\mathrm{C}+\mathrm{F}-, \mathrm{C}-\mathrm{F}+$, $\mathrm{C}-\mathrm{F}-$ ) between the distractor and the conjunctive targetdefining property was manipulated to reveal the separate effect of two ACSs, and the relative position (ThDv, TvDh, $\mathrm{ThDh}, \mathrm{TvDv}$ ) of the target and the distractor was manipulated to isolate the lateralized ERP activity elicited by the distractor from that elicited by the target. Moreover, different display durations (1,000 or $300 \mathrm{~ms}$ ) were assigned to two experiments to highlight the enhancement of target-relevant properties and the inhibition of target-irrelevant properties, respectively. The results suggest that fACS tends to enhance the processing of target-relevant properties for long display durations, whereas fACS inhibits the processing of target-irrelevant properties for short display durations. No obvious effects of cACS were found in this study, suggesting the critical role of fACS relative to cACS on attentional enhancement and inhibition.

The results of the two experiments revealed attentional enhancement and inhibition in the conjunction search task. In Experiment 1, a distractor-elicited N2pc component occurred when the distractor was feature-matched $(\mathrm{C}-\mathrm{F}+)$ and in the horizontal position ( $\mathrm{TvDh}$ ), indicating that fACS can enhance the processing of target-relevant properties. This result is consistent with a previous study and supports the concept of contingent attentional capture (Folk et al., 2008). In Experiment 2, a distractor-elicited Pd component was observed when the distractor was feature-mismatched $(\mathrm{C}+\mathrm{F}-)$ and in the horizontal position (TvDh), indicating that fACS can inhibit the processing of target-irrelevant properties. This result is consistent with a previous study (Sawaki et al., 2012; Hilimire et al., 2012) but extends the Pd component to conjunction search tasks. Notably, a previous study found that a Pd component was elicited by a memory-matching probe, indicating that attentional inhibition can occur for target-relevant properties, not target-irrelevant properties (Sawaki \& Luck, 2011). In this view, the Pd component observed in Experiment 2 might reflect inhibition of the target-relevant property rather than the target-irrelevant property. However, this interpretation could be excluded by the reliable Pd component observed under the F-C- condition, in which the distractor was fully target-irrelevant. Some inconsistent previous studies (Lien, Ruthruff, Goodin, \& Remington, 2008; Lien, Ruthruff, \& Cornett, 2010) reported an N2pc component, rather than a Pd component, elicited by short-display target-matching cues $(50 \mathrm{~ms})$, indicating that ACS can also enhance target-relevant properties within short durations. This finding could be interpreted by the hypothesis that "active inhibition would only be applied to distractors in trials in which they compete for attention with a simultaneously present target" (Kiss et al., 2013, p. 757). In this view, Lien et al. reported in previous studies that when the cue was not simultaneously presented with the target, active inhibition could not be triggered (Pd component).

The different lateralized ERP components observed in Experiment 1 and Experiment 2 might reveal the influence of display durations on enhancement and inhibition. Specifically, in Experiment 1, when the display durations were 1,000-ms long, the distractor elicited an N2pc component, indicative of attentional capture. In contrast, in Experiment 2, when display durations were limited to $300 \mathrm{~ms}$, the distractor elicited a Pd component, indicative of attentional inhibition. Kiss et al. (2012) and Sawaki and Luck (2010) found a similar pattern, and they postulated that the display duration might affect the time window for target detection, selection, and identification. When display durations were long enough to provide a broad time window, a salient distractor tends to capture attention via efficient searching. In contrast, when display durations were short, creating a narrow the time window, the distractor may not capture attention due to efficient searching. However, the previous study did not find a significant interaction across two experiments. One possibility is that the conjunction search task in the present study, in contrast to the singleton search task in the previous study (Kiss et al., 2012), might demand more attentional engagement and a broader time window to identify the target. As shown in Figures 2 and 4, even for long durations, the purely salient distractor (C-F-) did not elicit a reliable contralateral component. Consequently, mild enhancement and mild inhibition were observed in two experiments, but an interaction was not observed. Even so, the present study revealed a reliable distractor-elicited N2pc component and a reliable distractorelicited Pd component with different display durations, and these results indicated the different effects of ACS.

Another issue is the imbalanced sensory energy when one item occurred laterally ( $\mathrm{ThDv} \& \mathrm{TvDh}$ ), similar to the report by Hickey et al. (2009). In these conditions, the contralateral component (N2pc or Pd) plausibly reflects a sensory activity rather than attentional activity. As shown in the first rows of Figures 2, 3 , and 4, a reliable early contralateral ERP component was elicited by the lateralized target ( $\mathrm{ThDv}$ ) across all matching conditions $[t$ tests for mean amplitudes across the $100-200 \mathrm{~ms}$ time window. Exp. 1: C+F-, $t(14)=-2.86, p<0.005 ; \mathrm{C}-\mathrm{F}+$, $t(14)=-2.53, p<0.01$; C-F-, $t(14)=-4.68, p<0.001$; Exp. 2 : $\mathrm{C}+\mathrm{F}-, t(19)=-3.54, p<0.001 ; \mathrm{C}-\mathrm{F}+, t(19)=-3.12, p<0.005$; $\mathrm{C}-\mathrm{F}-, t(19)=-3.67, p<0.001]$. As shown in the second rows of Figures 2, 3, and 4, we also found a reliable early contralateral ERP component elicited by the lateralized distractor (TvDh) across all matching conditions [Exp. 1: C+F-, $t(14)=-2.57, p$ $<0.005 ; \mathrm{C}-\mathrm{F}+, t(14)=-4.28, p<0.001 ; \mathrm{C}-\mathrm{F}-, t(14)=-3.32, p<$ 0.001 ; Exp. 2 : C+F-, $t(19)=-2.89, p<0.005 ; \mathrm{C}-\mathrm{F}+, t(19)=$ $-3.44, p<0.001$; C-F-, $t(19)=-2.77, p<0.005]$ in the 100 - to 200 -ms time window post-stimulus onset $(150 \mathrm{~ms}$ at the peak in Figure 4). The lateralized stimuli seemed to elicit similar sensory responses in ERPs $(p s>0.11)$. Note that under the ThDv condition of Experiment 1 (first row in Figure 2 and upper left in 
Figure 4), the early laterality was larger for the C-F- condition relative to the $\mathrm{C}+\mathrm{F}$ - condition (difference $=0.66 \mu \mathrm{v}, p<0.005$ ), suggesting that a vertical distractor can affect the lateralized response to the target before the $\mathrm{N} 2 \mathrm{pc}$ component. This is probably because the early laterality elicited by the target is influenced by the salience of the target, and when the vertical distractor was C-F-, the high salience of the target could trigger a larger laterality. Moreover, later laterality during 220-280 ms in this condition was characterized by a similar pattern for the different matching conditions ( $\mathrm{C}-\mathrm{F}->\mathrm{C}+\mathrm{F}->\mathrm{C}-\mathrm{F}+)$, suggesting that more salient targets could trigger larger attentional allocation. In this case, both early (100-200 ms) and late (220-280 ms) laterality might be influenced by the salience of the target. Notably, this covariation only occurred when the vertical distractor affected the lateral target (ThDv) under long time durations $(1,000 \mathrm{~ms})$, and thus, that the late laterality elicited by the distractor (TvDh, the most concern in the present study) was not influenced by the early laterality. However, when the search display was balanced on each side (ThDh and TvDv), no contralateral component emerged ( $p s>0.25$, see the two bottom rows in Figures 2, 3, and 4), suggesting that the early contralateral components might reflect imbalanced sensory energy rather than the attentional capture.

Surprisingly, cACS did not obviously function in attentional enhancement and inhibition in the present study, in accordance with the results that the $\mathrm{Nt}$ and $\mathrm{Pd}$ components elicited by the $\mathrm{C}+\mathrm{F}$ - and $\mathrm{C}-\mathrm{F}$ - distractors were equivalent. The disappearance of cACS may be consistent with the view that semantic categories are doubtful attentional top-down sets (Wolfe \& Horowitz, 2004), and the reduction in cACS might be explained by the fact that activating an entire category composed of many diagnostic feature parts demands more resources (Reeder \& Peelen, 2013; Yang \& Zelinsky, 2009). However, several studies observed cACS in visual search tasks (Wyble et al., 2013; Wu et al., 2013; Wu et al., 2016). Wu et al. (2016) found an effect of cACS in a conjunction search task embedded in an RSVP stream. Leblanc et al. (2008) even found automatic activation of cACS in a task involving feature singleton search. The conflicting results between the present study and previous studies may be explained as follows. First, the task employed by Wu et al. (2016) was to recognize a target among 15 non-targets, and the properties of the non-targets were heterogeneous, forcing participants to adopt feature search mode (Bacon \& Egeth, 1994). Moreover, the nontargets used in the task in Leblanc et al. (2008) were always in the same category as the target, and the repeated occurrences of the target-relevant category could activate the effects of cACS. However, in the present study, the search array contained only one target and one distractor, without other non-targets that could amplify the effects of cACS. The effects of cACS might be mild in this conjunction search task and were not obvious. Second, Wu et al. (2016) found that cACS demands more attentional engagement to operate. In their study, when the target and a task-relevant distractor appeared simultaneously (insufficient engagement), the effects of cACS were eliminated or even disappeared, whereas when a task-relevant distractor occurred $160 \mathrm{~ms}$ before the target (sufficient engagement), cACS began to operate in the visual search. However, in the present study, the search array was composed of the target and a distractor that were presented simultaneously. cACS could be eliminated abruptly without sufficient engagement. Third, because the present study specifically referred fACS to color dimension and cACS to alphanumeric categories, the result that fACS operates more critically than cACS in the conjunction search task may not be a general conclusion. Some studies found greater weight allocated to the color dimension than to other feature dimensions (e.g., shape, size) (Kiss et al., 2013; Seiss, Kiss, \& Eimer, 2009), and additional categories using natural scenes (e.g., teddy bears, body parts, cars, etc.) have been investigated (Yang \& Zelinsky, 2009; Reeder \& Peelen, 2013). Therefore, further research should focus on the different roles of fACS and cACS in response to different types of dimensions in conjunction search tasks.

The present results also confirm the hypothesis that lateralized ERP activity is likely to be elicited by horizontal relative to vertical stimuli. The results were consistent in that no reliable contralateral ERP activity occurred when stimuli were presented at vertical positions (TvDv) in both experiments, which confirms the central findings of Hickey et al., (2009), who demonstrated that the lateralized ERP activity reflects the processing of horizontal stimuli. Moreover, a new test was conducted to investigate whether $\mathrm{Nt}$ and $\mathrm{Pd}$ are subcomponents of the N2pc component. The test factors included a hypothetical N2pc component, which was calculated by the absolute algebraic sum of the $\mathrm{Nt}$ (lateralized ERP in ThDv) and Pd (in TvDh) component, and an empirical N2pc, which was calculated by the absolute algebraic sum of the N2pc component (in $\mathrm{ThDh}$ ) and control (in TvDv). RANOVA including within-participant factors for the test $\mathrm{N} 2 \mathrm{pc}$ (hypothetical vs. empirical) and matching (C+F-, C-F+ , C-F-) and a between-participant factor for experiment (Exp. 1 vs. Exp. 2) revealed no significant main effects or interactions ( $p s>0.12$ ), suggesting similar amplitudes of the lateral component between the hypothetical and empirical N2pc. This result provides some evidence supporting the hypothesis that the N2pc component reflects the summation of the Nt and Pd components (Hickey et al., 2009).

In summary, the current study provides new evidence for the different roles of $\mathrm{CACS}$ and fACS in attentional enhancement and inhibition in conjunction search tasks. An N2pc indicative of attentional capture was elicited by lateral distractors with long display durations in Experiment 1, suggesting that ACS can enhance the processing of targetrelevant properties. In contrast, a Pd indicative of attentional inhibition was elicited by lateral distractors with short display 
durations in Experiment 2, suggesting that ACS can inhibit the processing of target-irrelevant properties. Moreover, fACS operates more critically relative to cACS in both enhancing and inhibiting attention, suggesting that differences exist between the two ACSs in conjunction search tasks.

Acknowledgements This research was supported by the National Natural Science Foundation of China under Grant (31371142) and the Initiative Scientific Research Program, Tsinghua University, China under Grant (2011Z02177) to SF.

\section{References}

Adamo, M., Pun, C., \& Ferber, S. (2010). Multiple attentional control settings influence late attentional selection but do not provide an early attentional filter. Cognitive Neuroscience, 1, 102-110.

Atchley, P., Kramer, A. F., \& Hillstrom, A. P. (2000). Contingent capture for onsets and offsets: Attentional sets for perceptual transients. Journal of Experimental Psychology: Human Perception and Performance, 26, 594-606.

Bacon, W. F., \& Egeth, H. E. (1994). Overriding stimulus-driven attentional capture. Perception \& Psychophysics, 55, 485-496.

Eimer, M. (1996). The N2pc component as an indicator of attentional selectivity. Electroencephalography and Clinical Neurophysiology, 99, 225-234.

Folk, C. L., Leber, A. B., \& Egeth, H. E. (2008). Top-down control settings and the attentional blink: Evidence for non-spatial contingent capture. Visual Cognition, 16, 616-642.

Folk, C. L., Remington, R. W., \& Johnston, J. C. (1992). Involuntary covert orienting is contingent on attentional control settings. Journal of Experimental Psychology: Human Perception and Performance, 18, 1030-1044.

Folk, C. L., Remington, R. W., \& Wright, J. H. (1994). The structure of attentional control: Contingent attentional capture by apparent motion, abrupt onset, and color. Journal of Experimental Psychology: Human Perception and Performance, 20, 317-329.

Hickey, C., Di Lollo, V., \& McDonald, J. J. (2009). Electrophysiological indices of target and distractor processing in visual search. Journal of Cognitive Neuroscience, 21(4), 760-775.

Hickey, C., Mcdonald, J. J., \& Theeuwes, J. (2006). Electrophysiological evidence of the capture of visual attention. Journal of Cognitive Neuroscience, 18(4), 604-613.

Hilimire, M. R., Hickey, C., \& Corballis, P. M. (2012). Target resolution in visual search involves the direct suppression of distractors: Evidence from electrophysiology. Psychophysiology, 49, 504-509.

Irons, J. L., Folk, C. L., \& Remington, R. W. (2012). All set! Evidence of simultaneous attentional control settings for multiple target colors. Journal of Experimental Psychology: Human Perception and Performance, 38(3), 758.

Kiss, M., Grubert, A., Petersen, A., \& Eimer, M. (2012). Attentional capture by salient distractors during visual search is determined by temporal task demands. Journal of Cognitive Neuroscience, 24(3), 749-759.

Kiss, M., Grubert, A., \& Eimer, M. (2013). Top-down task sets for combined features: Behavioral and electrophysiological evidence for two stages in attentional object selection. Attention, Perception, and Psychophysics, 75(2), 216-228.

Leblanc, E., Prime, D. J., \& Jolicoeur, P. (2008). Tracking the location of visuospatial attention in a contingent capture paradigm. Journal of Cognitive Neuroscience, 20(4), 657-671.

Lien, M. C., Ruthruff, E., Goodin, Z., \& Remington, R. W. (2008). Contingent attentional capture by top-down control settings:
Converging evidence from event-related potentials. Journal of Experimental Psychology: Human Perception and Performance, 34(3), 509.

Lien, M. C., Ruthruff, E., \& Cornett, L. (2010). Attentional capture by singletons is contingent on top-down control settings: Evidence from electrophysiological measures. Visual Cognition, 18(5), 682-727.

Luck, S. J., Girelli, M., McDermott, M. T., \& Ford, M. A. (1997). Bridging the gap between monkey neurophysiology and human perception: An ambiguity resolution theory of visual selective attention. Cognitive Psychology, 33, 64-87.

Luck, S. J., \& Hillyard, S. A. (1994). Spatial filtering during visual search: Evidence from human electrophysiology. Journal of Experimental Psychology: Human Perception \& Performance, 20, 1000-1014.

Mazza, V., Turatto, M., \& Caramazza, A. (2009). Attention selection, distractor suppression and N2pc. Cortex, 45, 879-890.

Moore, K. S., \& Weissman, D. H. (2010). Involuntary transfer of a topdown attentional set into the focus of attention: Evidence from a contingent attentional capture paradigm. Attention, Perception, \& Psychophysics, 72(6), 1495-1509.

Reeder, R. R., \& Peelen, M. V. (2013). The contents of the search template for category-level search in natural scenes. Journal of Vision, 13(3), 13.

Sawaki, R., \& Luck, S. J. (2010). Capture versus suppression of attention by salient singletons: Electrophysiological evidence for an automatic attend-to-me signal. Attention, Perception, and Psychophysics, 72(6), 1455-1470.

Sawaki, R., \& Luck, S. J. (2011). Active suppression of distractors that match the contents of visual working memory. Vision Cognition, 19(7), 956-972.

Sawaki, R., Geng, J. J., \& Luck, S. J. (2012). A common neural mechanism for preventing and terminating the allocation of attention. Journal of Neuroscience, 32(31).

Seiss, E., Kiss, M., \& Eimer, M. (2009). Does focused endogenous attention prevent attentional capture in pop-out visual search? Psychophysiology, 46, 703-717.

Serences, J. T., Shomstein, S., Leber, A. B., Golay, X., Egeth, H. E., \& Yantis, S. (2005). Coordination of voluntary and stimulus-driven attentional control in human cortex. Psychological Science, 16(2), $114-122$.

Theeuwes, J. (1991). Cross-dimensional perceptual selectivity. Perception \& Psychophysics, 50, 184-193.

Theeuwes, J. (2010). Top-down and bottom-up control of visual selection. Acta Psychologica, 135, 77-99.

Wolfe, J. M., \& Horowitz, T. S. (2004). What attributes guide the deployment of visual attention and how do they do it? Nature Reviews Neuroscience, 5(6), 495-501.

Woodman, G. F., \& Luck, S. J. (2003). Serial deployment of attention during visual search. Journal of Experimental Psychology: Human Perception and Performance, 29, 121-138.

Wu, X., Liu, X., \& Fu, S. (2016). Feature- and category-specific attentional control settings are differently affected by attentional engagement in contingent attentional capture. Biological Psychology, 118, 8-16.

Wu, R., Scerif, G., Aslin, R. N., Smith, T. J., Nako, R., \& Eimer, M. (2013). Searching for something familiar or novel: Top-down attentional selection of specific items or object categories. Journal of Cognitive Neuroscience, 25(5), 719-729.

Wyble, B., Folk, C., \& Potter, M. C. (2013). Contingent attentional capture by conceptually relevant images. Journal of Experimental Psychology: Human Perception and Performance, 39(3), 861.

Yang, H., \& Zelinsky, G. J. (2009). Visual search is guided to categorically defined targets. Vision Research, 49, 2095-2103.

Yantis, S., \& Jonides, J. (1984). Abrupt visual onsets and selective attention: Evidence from visual search. Journal of Experimental Psychology: Human Perception and Performance, 10, 601-621. 